\title{
A continuous flow multi-laminar micromixer with improved flow profile for infrared analysis of chemical reaction kinetics
}

\author{
Wolfgang Buchegger ${ }^{1}$, Christoph Wagner ${ }^{2}$, Bernhard Lendl ${ }^{2}$, M.Kraft $^{3}$, Michael Vellekoop ${ }^{1}$ \\ ${ }^{1}$ Institute of Sensor and Actuator Systems \\ ${ }^{2}$ Institute of Chemical Technologies and Analytics \\ Vienna University of Technology \\ Gusshausstrasse 27-29/E366, 1040 Wien \\ ${ }^{3}$ CTR Carinthian Tech Research AG, Villach
}

\begin{abstract}
A continuous flow micromixer is utilized for time resolved infrared spectroscopy to investigate (bio) chemical reaction kinetics. This passive multi-lamination mixer was optimized for millisecond mixing using low flow rates by fluid dynamic simulations. Four lamination layers in the mixing channel yield a reduction of the diffusion length and decrease mixing time significantly. Laser scanning confocal measurements enabled to visualize the individual fluid layers and comparing them to simulation results. An infrared measurement of the sulfite-formaldehyde acid-to-alkali clock reaction of sodium sulfite and formaldehyde solution was carried out to characterize this continuous flow micromixer.
\end{abstract}

\section{Keywords}

Microfluidic Mixing, Passive Micromixer, Infrared Spectroscopy, Kinetics

\section{Introduction}

Microfluidic technology provides great opportunities as a tool for modern analytical analysis. The physical laws, such as diffusion length, small volume, present at micro scale enable to speed up detection methods, reduce sample consumption and increase sensitivity. Often, an optical detection system is applied utilizing a broad wavelength region ranging from UV to visible and infrared light. Fluorescencebased methods are attractive as they provide excellent selectivity [1]. However, labeling steps are necessary and hence increase the effort and cost for the measurement. Therefore, a rising interest in a label free detection method exists. Label-free detection techniques applicable for microfluidics include Raman spectroscopy [2] and infrared spectroscopy, in particular time resolved Fourier transform infrared (TR-FTIR) spectroscopy. Recently, chemical reaction kinetic measurements, applying this technique in combination with micromixers have been presented [3]. When analyzing aqueous solutions by IR spectroscopy the high absorption of water has a significant influence on the measurement signal. To achieve a good signal strength the optical path and thereby the channel height through the aqueous medium should not exceed 8 to $30 \mu \mathrm{m}$ [4]. Our micromixer design generates four laminated fluid layers out of two fluid feeds by a distribution network in a mixing channel with a cross section area of 8 by $200 \mu \mathrm{m}^{2}$, see Fig.1a). Using a 2D simulation model of the microstructure (Fig. 1b) the individual fluid layers in the mixing channel can be visualized. The fluid layers differ in their thickness to reduce the mixing time. The outer fluid layers only have one diffusion face (upwards or downwards) whereas the inner layers diffuse in both directions. By optimizing these fluid layers the diffusion length and therefore the mixing time can be reduced by a factor of $9\left(t_{\text {mix }} \sim d_{\text {Diff }}^{2}\right)$ compared to a two layer system [5]. In our system the mixing channel also serves as measurement area for the IR measurement. Measuring closer to the beginning of the mixing channel corresponds to an earlier time in the reaction than moving the measurement spot further down the channel. The rectangular shaped measurement spots are placed next to each other resulting in a certain time resolution. This time resolution can be adapted by varying the pumping speed of the fluid. Increasing the pumping speed results in a higher time resolution but in a shorter observation window for the measurement as the mixing channel ends after about $9 \mathrm{~mm}$. Proper mixing before the IR measurement is crucial to get meaningful results. Therefore only chemical reactions with reaction times one magnitude higher than the mixing time can be investigated. 
a)

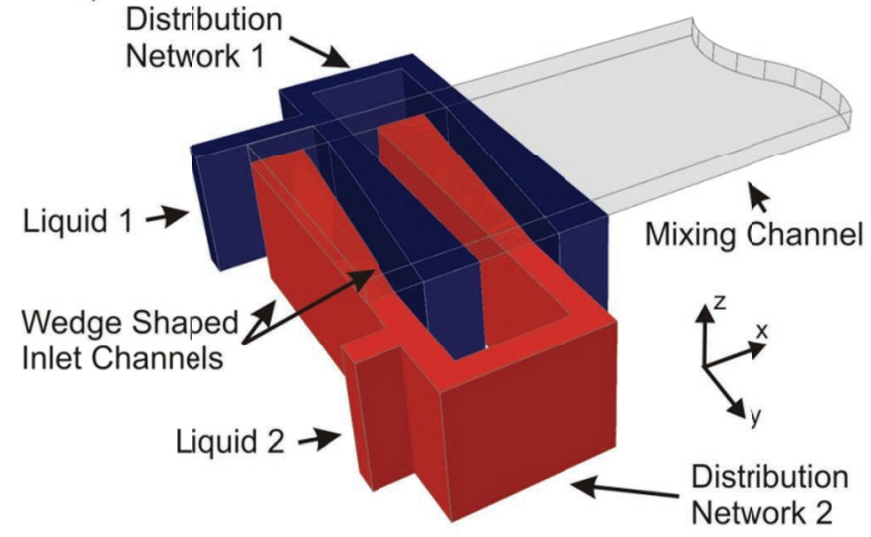

b)

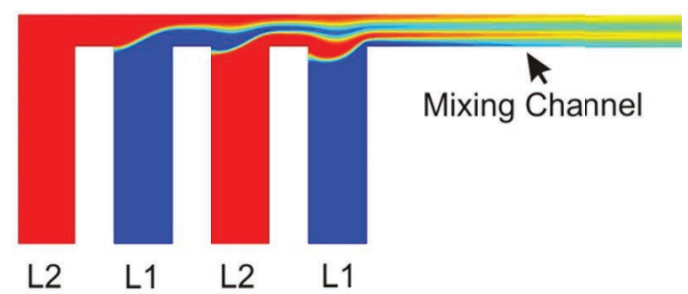

Figure 1. Schematic of the lamination micromixer with two sided fed, wedge shaped inlet channels, distribution network and mixing channel (a) and a $2 \mathrm{~d}$ cross sectional view of a simulation of the micromixer (b).

\section{Simulation}

For the design geometry of the distribution network, responsible for the formation of the individual fluid layers, and the optimization of the inlet channel shape, computational fluid dynamic (CFD) simulations with Comsol Multiphysics were performed. For the flow simulation and Incompressible Navier Stokes Model was applied whereas for the concentration plots a Convection and Diffusion Model was used. Further, physical parameters of water representing the chemical solutions used in the experiments and a concentration of $0 \mu$ molar for one ( $100 \%$ L1) and $100 \mu$ molar for the second reagent ( $100 \%$ L2) were set. In Fig. 2 simulation results of a rectangular inlet design is compared to a wedged inlet design is compared. The cross section of a concentration plot right after the inlet channels $A$ and $2 \mathrm{~ms}$ further down the mixing channel $B$ are shown in the image. If the concentration plot of the two designs is compared at position $\mathrm{A}$ a non-uniform horizontal distribution of reagents can be seen for the straight inlet design whereas the wedged design shows distinct horizontal fluid layers. The irregular fluid llayers occurring in the rectangular shaped mixer originate in a pressure drop along the inlet channel. By widening up the inlet channel to a wedge form this pressure drop can be compensated and hence a straight layer can be formed in the mixing channel. If we look at the cross section plot $2 \mathrm{~ms}$ later, further down the mixing channel the mixing efficiency of the two designs can be compared. Clearly, the performance of the wedged design is much better as a homogenous mix of both reagents is present in the mixing channel whereas the rectangular inlet channel shows a non-uniform distribution of reagents. With an estimated diffusion coefficient of $D=2 \times 10^{-9} \mathrm{~m}^{2} / \mathrm{s}$ a mixing time of about $t_{\mathrm{min}}=1 \mathrm{~ms}$ was calculated.

\section{Measurements}

In a first measurement the individual fluid layers were visualized. As the mixing channel is observed from top there is no opportunity to see the layers by optical microscopy. Therefore optical laser scanning confocal measurements were carried out to visualize the horizontal fluid layers proving simulated results. A Nikon C80i microscope with a 20 fold objective was used for the experiment. We utilized a laser with a wavelength of $488 \mathrm{~nm}$ for excitation and a filter with $515 \mathrm{~nm}$ and a bandwidth of $30 \mathrm{~nm}$ for detection. The two liquids, deionized (DI)-water and a $5 \mu$ molar fluorescein-DI-water solution were pumped through the micromixer using pressure driven syringe pumps. Fig. 3a) shows the result of a cross section measurement of the mixing channel with $200 \mu \mathrm{m}$ width. Four fluid layers are formed right after the inlet channels. As predicted by simulations straight inlet channels lead to poor layer formation (Fig. 3a). Four homogenous fluid layers are only formed with wedged inlet channels (Fig. 3c). This is of major importance for time resolved measurements as mixing time is reduced enabling investigating faster chemical reactions. 

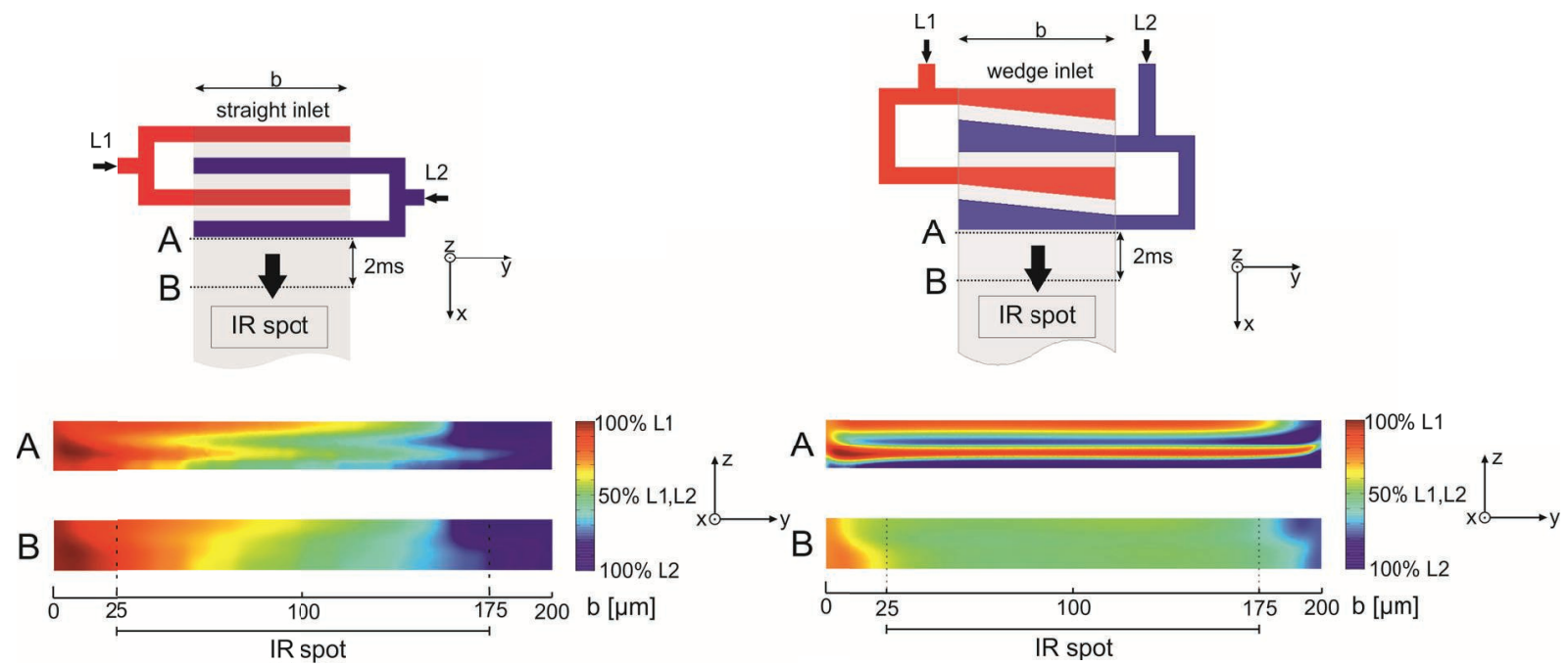

Figure 2. CFD simulation of the micromixer with straight inlet channels on the left and with wedged inlet channels on the right. The cross section area is $8 \mu \mathrm{m} \times 200 \mu \mathrm{m}$ in size and shows the concentration profile at A (all four developed fluid layers) and B ( $2 \mathrm{~ms}$ after fluid layers were developed). The simulations suggest that a wedged inlet channel increases the mixing ability tremendously.

In a second experiment the infrared measurement of the sulfite-formaldehyde acid-to-alkali clock reaction of sodium sulfite $\mathrm{Na}_{2} \mathrm{SO}_{3}$ and formaldehyde solution $\mathrm{HCHO}$ was carried out, serving as a system characterization measurement of this infrared continuous flow mixer. All together 86 spectra were recorded along the mixing channel, each corresponding to a certain reaction time. After the spectra were recorded a three-dimensional plot was created containing the kinetic information of the chemical reaction, Fig.4c). The absorption band of the sulfite ion at $942 \mathrm{~cm}^{-1}$ decreases concurrently with an increase of the product band of hydroxymethane sulfonate $\mathrm{CH}_{2}(\mathrm{OH}) \mathrm{SO}_{3}{ }^{-}$at $1180 \mathrm{~cm}^{-1}$. The formalin band at $1025 \mathrm{~cm}^{-1}$ exhibits a split into two bands during the reaction, a phenomenon that is attributed to a band overlap caused by the $\mathrm{C}-\mathrm{O}$ stretching vibration and the symmetric stretching vibration of $\mathrm{SO}_{2}$ moiety of the product. The lower picture shows a direct comparison of the first and the last of the 86 recorded spectra; the arrows indicate the wavenumbers of the changing reagents. Compared to an earlier mixer generation the mixing time could be improved by a factor of $\sim 25$, [6].

a) straight inlet

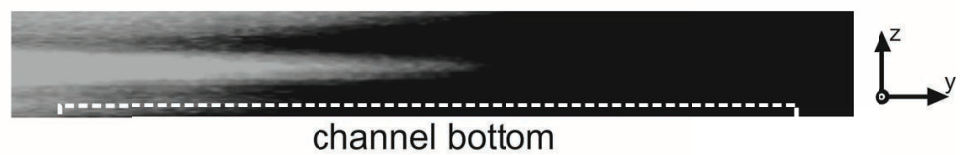

b)

wedge inlet

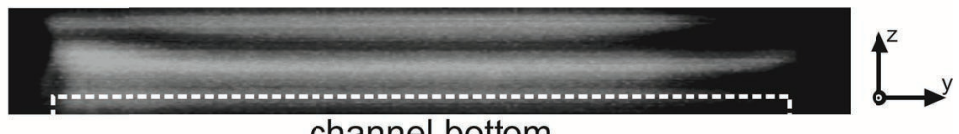

channel bottom

Figure 3. Laser scanning confocal measurement of the fluid layers in the micromixer of straight channel design (a) and optimized wedge shaped design (b). 

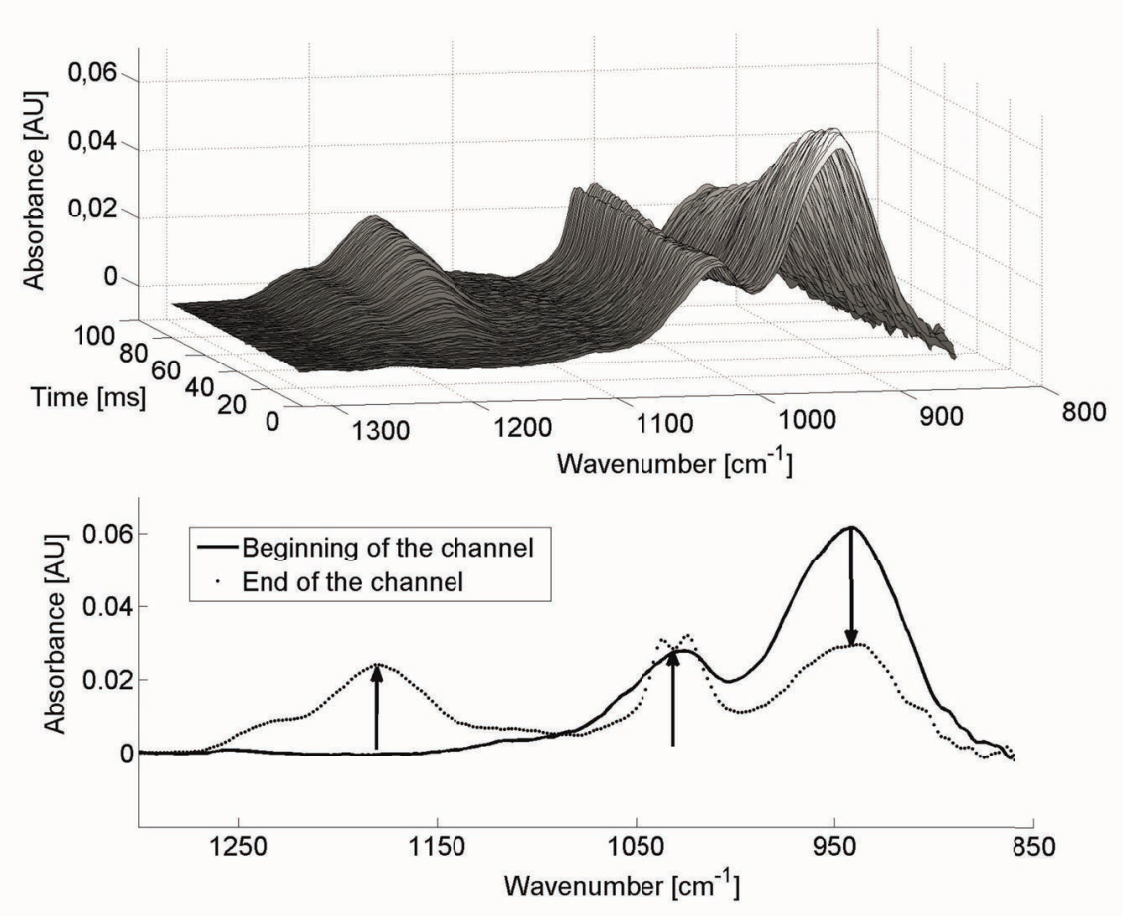

Figure 4. A TR-FTIR measurement of the clock reaction between $\mathrm{Na}_{2} \mathrm{SO}_{3}$ and $\mathrm{HCHO}$ solution is depicted. The upper plot shows IR absorbance spectra at different measurement times; the lower plot shows a comparison of the initial and the ffinal spectra of the measurement.

\section{Conclusion}

Wedge shaped inlet channels form homogeous fluid layers in the channel and hence, reduce the diffusion length and the mixing time compared to rectangular mixing channels. Laminar fluid layers formed in our micromixer could be made visible by confocal laser scanning microscopy and support the simulation results. A mixing time of $1 \mathrm{~ms}$ enabes to investigate a wide range of chemical reactions by IR spectroscopy and therefore provides and effective and label free option of determining kinetics of chemical reactions compared to similar fluorescent based techniques. The time resolved measurement of the sulfite-formaldehyde acid-to-alkali clock reaction shows first IR measurement results with an excellent singal to noise ratio in the mid infrared range. Micromixers are of great potential for analytical analysis of various reaction kinetics and are of potential interest for fast and reliable analysis of enzymatic reactions with low cost and litte sample amount.

\section{Acknowledgments}

For the sensor fabrication and the technical support we thank E. Svasek, P. Svasek from the ISAS Technology Lab at the Vienna University of Technology. For financial support we gratefully acknowledge the COMET Competence Center Program of the Austrian Government.

\section{References}

[1] S. T. Hess et al., Biological and Chemical Applications of Fluorescence Correlation Spectroscopy: A Review, Biochemistry (2002), 41, 3, 697-705

[2] S.-A. Leung et al., A method for rapid reaction optimization in continuous-flow microfluidic reactors using online Raman spectroscopic detection, Analyst (2005), 130, 46-51 
[3] C. Wagner et al., Time-resolved mid-IR spectroscopy of (bio) chemical reactions in solution utilizing a new generation of continuous-flow micro-mixers, AnalBioanalChem, DOI10.1007/s00216-010-4643-2

[4] J.K. Koenig et al., Analytical Applications of FT-IR to Molecular and Biological Systems (1980), 241255, D. Reidel, Boston

[5] W. Buchegger et al., A highly uniform lamination micromixer with wedge shaped inlet channels for time resolved infrared spectroscopy (2010), MicroNanofluid, DOI: 10.1007/s10404-010-0722-0

[6] N. Kaun, M.J. Vellekoop, B. Lendl, Time-resolved Fourier transform infrared spectroscopy of chemical reactions in solution using a focal plane array detector, App/Spectrosc., 2006, 60, 1273-1278 